УДК 515.1

\author{
A. Mukharjee, A. Roy Choudhury
}

\title{
ON BONDING OF SOME BITOPOLOGICAL NOTIONS
}

\begin{abstract}
A. Mukharjee, A. Roy Choudhury. On bonding of some bitopological notions, Mat. Stud. 48 (2017), 108-112.

In this paper, we study two strong forms of separations axioms on bitopological spaces and we observe that the results on such axioms are odd compared to prevailing results of bitopological spaces or topological spaces, e.g. we see that $Y \subset X$ need not be $\left(\mathscr{P}_{i}\right)$ closed for any $i \in\{1,2\}$ to be the relative bitopological space $\left(Y, \mathscr{P}_{1 Y}, \mathscr{P}_{2 Y}\right)$ strongly pairwise normal under some conditions including the bitopological space $\left(X, \mathscr{P}_{1}, \mathscr{P}_{2}\right)$ to be strongly pairwise normal.
\end{abstract}

1. Introduction. Kelly ([2]) introduced the notion of bitopological spaces on noticing that there exist two topologies on a quasi-metric space due to asymmetric nature of quasi-metric spaces. According to Kelly ([2]), a bitopological space is a nonempty set $X$ endowed with two topologies $\mathscr{P}_{1}$ and $\mathscr{P}_{2}$, and it is denoted by $\left(X, \mathscr{P}_{1}, \mathscr{P}_{2}\right)$. Since the introduction of the notion of bitopological spaces by Kelly ([2]), bitopological settings are enriching by means of many diverse and worthy contributions like pairwise compactness, pairwise connectedness, pairwise paracompactness, various forms of separation axioms, etc. It is fact that the results obtained in the field of bitopological spaces are mainly generalizations of the results of topological spaces. For any $\left(\mathscr{P}_{i}\right)$ open set $U$ of a bitopological space $\left(X, \mathscr{P}_{1}, \mathscr{P}_{2}\right)$, the trivial result $\left(\mathscr{P}_{j}\right) \operatorname{Int}(U) \subset U,(i, j \in\{1,2\}, i \neq j)$ motivates us $([3])$ to introduce notions of some strong separation axioms and strong compactness in bitopological spaces. With the concepts introduced in [3], we not only study the properties of a bitopological space $\left(X, \mathscr{P}_{1}, \mathscr{P}_{2}\right)$ but also properties of both topological spaces $\left(X, \mathscr{P}_{1}\right)$ and $\left(X, \mathscr{P}_{2}\right)$ simultaneously, i.e. to say that in [3], we mixed up the properties of a bitopological space $\left(X, \mathscr{P}_{1}, \mathscr{P}_{2}\right)$ with the properties of both topological spaces $\left(X, \mathscr{P}_{1}\right)$ and $\left(X, \mathscr{P}_{2}\right)$. Naturally, the results obtained in [3] as well as in the present paper are odd compared to prevailing results of bitopological spaces or topological spaces. For example, in Theorem 6, we see that $Y \subset X$ need not be $\left(\mathscr{P}_{i}\right)$ closed for any $i \in\{1,2\}$ to be the relative bitopological space $\left(Y, \mathscr{P}_{1 Y}, \mathscr{P}_{2 Y}\right)$ strongly pairwise normal under some conditions when the bitopological space $\left(X, \mathscr{P}_{1}, \mathscr{P}_{2}\right)$ is strongly pairwise normal. But in topological spaces, closed subspaces of normal spaces are normal.

Throughout the paper, a bitopological space $\left(X, \mathscr{P}_{1}, \mathscr{P}_{2}\right)$ is simply denoted by $X . \mathbb{R}$ denotes the set of real numbers and $\mathbb{N}$, the set of natural numbers. $(\mathscr{T}) \operatorname{Int}(A)$ denotes the interior and $(\mathscr{T}) \mathrm{Cl}(A)$, the closure of a set $A$ in the topological space $(X, \mathscr{T})$. For a topological space $(X, \mathscr{T})$ and $A \subset X$, we write $\left(A, \mathscr{T}_{A}\right)$ to denote the subspace on $A$ of $(X, \mathscr{T})$. So the relative

2010 Mathematics Subject Classification: 54E55.

Keywords: pairwise regular; pairwise normal; strongly pairwise regular; strongly pairwise normal. doi:10.15330/ms.48.1.108-112 
bitopological space for $\left(X, \mathscr{P}_{1}, \mathscr{P}_{2}\right)$ corresponding to $Y \subset X$ is $\left(Y, \mathscr{P}_{1 Y}, \mathscr{P}_{2 Y}\right)$. Always $i, j \in\{1,2\}$ and whenever $i, j$ appear together, $j \neq i$.

The following known definitions are recalled to make the article self-contained.

Definition 1 (Kelly, [2]). In a bitopological space $X, \mathscr{P}_{i}$ is said to be regular with respect to $\mathscr{P}_{j}$ if for each $x \in X$ and each $\left(\mathscr{P}_{i}\right)$ closed set $A$ with $x \notin A$, there exist $U \in \mathscr{P}_{i}$ and $V \in \mathscr{P}_{j}$ such that $x \in U, A \subset V$ and $U \cap V=\varnothing$. $X$ is said to be pairwise regular if $\mathscr{P}_{i}$ is regular with respect to $\mathscr{P}_{j}, i, j \in\{1,2\}, i \neq j$.

Definition 2 (Kelly, [2]). A bitopological space $X$ is said to be pairwise normal if for any pair of a $\left(\mathscr{P}_{i}\right)$ closed set $A$ and a $\left(\mathscr{P}_{j}\right)$ closed set $B$ with $A \cap B=\varnothing$, there exist $U \in \mathscr{P}_{j}$ and $V \in \mathscr{P}_{i}$ such that $A \subset U, B \subset V$ and $U \cap V=\varnothing$.

Definition 3 (Mukharjee, [3]). A bitopological space $X$ is said to be strongly pairwise regular if for each $x \in X$ and each $\left(\mathscr{P}_{i}\right)$ closed set $F$ with $x \notin F$, there exist a $\left(\mathscr{P}_{i}\right)$ open set $U$ and a $\left(\mathscr{P}_{j}\right)$ open set $V$ such that $x \in U, F \subset\left(\mathscr{P}_{i}\right) \operatorname{int}(V)$ and $U \cap V=\varnothing$.

Definition 4 (Mukharjee, [3]). A subset $A$ of a bitopological space $X$ is called $\left(\mathscr{P}_{i}, \mathscr{P}_{j}\right)$ dually open if there exists a $\left(\mathscr{P}_{j}\right)$ open set $U$ such that $A=\left(\mathscr{P}_{i}\right) \operatorname{Int}(U)$.

2. Bitopological strong separation axioms. Firstly, we obtain some properties on strongly pairwise regular spaces.

Theorem 1. For a bitopological space $\left(X, \mathscr{P}_{1}, \mathscr{P}_{2}\right)$, the following are equivalent.

(i) $X$ is strongly pairwise regular.

(ii) For each $x \in X$ and each $\left(\mathscr{P}_{i}\right)$ open set $U$ with $x \in U$, there exists a $\left(\mathscr{P}_{j}\right)$ closed set $E$ $(i, j \in\{1,2\}, j \neq i)$, such that $x \in\left(\mathscr{P}_{i}\right) \operatorname{Int}(E)$ and $\left(\mathscr{P}_{i}\right) \mathrm{Cl}(E) \subset U$.

(iii) For each $x \in X$ and each $\left(\mathscr{P}_{i}\right)$ open set $U$ with $x \in U$, there exists a $\left(\mathscr{P}_{i}\right)$ open set $G$ such that $x \in G \subset\left(\mathscr{P}_{i}\right) \mathrm{Cl}\left(\left(\mathscr{P}_{j}\right) \mathrm{Cl}(G)\right) \subset U,(i, j \in\{1,2\}, j \neq i)$.

Proof. (i) $\Rightarrow\left(\right.$ ii): Let $x \in X$ and $U$ be $\left(\mathscr{P}_{i}\right)$ open in $X$ such that $x \in U$. By strong pairwise regularity of $X$, we obtain a $\left(\mathscr{P}_{i}\right)$ open set $G$ and a $\left(\mathscr{P}_{j}\right)$ open set $H$ such that $x \in G, X-$ $U \subset\left(\mathscr{P}_{i}\right) \operatorname{Int}(H)$ and $G \cap H=\varnothing$. Since $G$ is $\left(\mathscr{P}_{i}\right)$ open set, $G \cap H=\varnothing$ implies that $G \subset\left(\mathscr{P}_{i}\right) \operatorname{Int}(X-H) \subset X-\left(\mathscr{P}_{i}\right) \mathrm{Cl}(H) \subset X-H$. Thus we get

$$
\begin{aligned}
& x \in G \subset\left(\mathscr{P}_{i}\right) \operatorname{Int}(X-H) \subset X-H \subset X-\left(\mathscr{P}_{i}\right) \operatorname{Int}(H) \subset U \\
& \text { since } X-U \subset\left(\mathscr{P}_{i}\right) \operatorname{Int}(H), \\
& \Rightarrow \quad x \in G \subset\left(\mathscr{P}_{i}\right) \operatorname{Int}(X-H) \subset X-H \subset\left(\mathscr{P}_{i}\right) \mathrm{Cl}(X-H) \subset U .
\end{aligned}
$$

We see that $E=X-H$ is a $\left(\mathscr{P}_{j}\right)$ closed set such that $x \in\left(\mathscr{P}_{i}\right) \operatorname{Int}(E)$ and $\left(\mathscr{P}_{i}\right) \mathrm{Cl}(E) \subset U$. (ii) $\Rightarrow\left(\right.$ iii): Let $x \in X$ and $U$ be $\left(\mathscr{P}_{i}\right)$ open in $X$ such that $x \in U$. By (ii), we obtain a $\left(\mathscr{P}_{j}\right)$ closed set $E$ such that $x \in\left(\mathscr{P}_{i}\right) \operatorname{Int}(E) \subset E \subset\left(\mathscr{P}_{i}\right) \mathrm{Cl}(E) \subset U$. Putting $G=$ $\left(\mathscr{P}_{i}\right) \operatorname{Int}(E)$, we see that $x \in G$ and

$$
\begin{aligned}
& \left(\mathscr{P}_{j}\right) \mathrm{Cl}(G) \subset E \subset\left(\mathscr{P}_{i}\right) \mathrm{Cl}(E) \subset U \text { as } E \text { is }\left(\mathscr{P}_{j}\right) \text { closed } \\
\Rightarrow \quad & \left(\mathscr{P}_{i}\right) \mathrm{Cl}\left(\left(\mathscr{P}_{j}\right) \mathrm{Cl}(G)\right) \subset\left(\mathscr{P}_{i}\right) \mathrm{Cl}(E) \subset U .
\end{aligned}
$$

So we get a $\left(\mathscr{P}_{i}\right)$ open set $G$ such that $x \in G \subset\left(\mathscr{P}_{i}\right) \mathrm{Cl}\left(\left(\mathscr{P}_{j}\right) \mathrm{Cl}(G)\right) \subset U$.

(iii) $\Rightarrow$ (i): Let $x \in X$ and $F$ be $\left(\mathscr{P}_{i}\right)$ closed with $x \notin F$. By (iii), we obtain a $\left(\mathscr{P}_{i}\right)$ open set $G$ such that $x \in G \subset\left(\mathscr{P}_{i}\right) \mathrm{Cl}\left(\left(\mathscr{P}_{j}\right) \mathrm{Cl}(G)\right) \subset X-F$. Now $\left(\mathscr{P}_{i}\right) \mathrm{Cl}\left(\left(\mathscr{P}_{j}\right) \mathrm{Cl}(G)\right) \subset X-F$ implies that $F \subset\left(\mathscr{P}_{i}\right) \operatorname{Int}\left(X-\left(\mathscr{P}_{j}\right) \mathrm{Cl}(G)\right)$. 
Theorem 2. If $\left(X, \mathscr{P}_{1}, \mathscr{P}_{2}\right)$ is a bitopological space such that $X$ is pairwise regular, $\left(X, \mathscr{P}_{i}\right)$ is regular for each $i \in\{1,2\}$ and either $\mathscr{P}_{1} \subset \mathscr{P}_{2}$ or $\mathscr{P}_{2} \subset \mathscr{P}_{1}$, then $X$ is strongly pairwise regular.

Proof. We suppose that $\mathscr{P}_{i} \subset \mathscr{P}_{j}$ for some $i, j \in\{1,2\}, i \neq j$. The following two cases may arise.

Case I: Let $x \in X$ and $F$ be a $\left(\mathscr{P}_{i}\right)$ closed set such that $x \notin F$. By the regularity of $\left(X, \mathscr{P}_{i}\right)$, there exist $\left(\mathscr{P}_{i}\right)$ open sets $U_{1}, V_{1}$ such that $x \in U_{1}, F \subset V_{1}$ and $U_{1} \cap V_{1}=\varnothing$. Since $\mathscr{P}_{i} \subset \mathscr{P}_{j}, V_{1}$ is also $\left(\mathscr{P}_{j}\right)$ open. So we can now say that there exists a $\left(\mathscr{P}_{i}\right)$ open set $U_{1}$ and a $\left(\mathscr{P}_{j}\right)$ open set $V_{1}$ such that $x \in U_{1}, F \subset\left(\mathscr{P}_{i}\right) \operatorname{Int}\left(V_{1}\right)$ and $U_{1} \cap V_{1}=\varnothing$.

Case II: Let $x \in X$ and $F$ be a $\left(\mathscr{P}_{j}\right)$ closed set such that $x \notin F$. By the pairwise regularity of $X$, there exist a $\left(\mathscr{P}_{j}\right)$ open set $U_{2}$ and a $\left(\mathscr{P}_{i}\right)$ open set $V_{2}$ such that $x \in U_{2}, F \subset V_{2}$ and $U_{2} \cap V_{2}=\varnothing$. Since $\mathscr{P}_{i} \subset \mathscr{P}_{j}, V_{2}$ is also $\left(\mathscr{P}_{j}\right)$ open. So we can now say that there exists a $\left(\mathscr{P}_{j}\right)$ open set $U_{2}$ and a $\left(\mathscr{P}_{i}\right)$ open set $V_{2}$ such that $x \in U_{2}, F \subset\left(\mathscr{P}_{j}\right) \operatorname{Int}\left(V_{2}\right)$ and $U_{2} \cap V_{2}=\varnothing$.

We now recall the following.

Definition 5 (Mukharjee, [3]). A bitopological space $X$ is said to be strongly pairwise normal if for each $\left(\mathscr{P}_{i}\right)$ closed set $E$ and each $\left(\mathscr{P}_{j}\right)$ closed set $F$ with $E \cap F=\varnothing$, there exist a $\left(\mathscr{P}_{j}\right)$ open set $U$ and a $\left(\mathscr{P}_{i}\right)$ open set $V$ such that $E \subset\left(\mathscr{P}_{i}\right) \operatorname{Int}(U), F \subset\left(\mathscr{P}_{j}\right) \operatorname{Int}(V)$ and $U \cap V=\varnothing$.

In [3], we noted down that if a bitopological space $X$ is strongly pairwise normal then the space $\left(X, \mathscr{P}_{i}\right)(i \in\{1,2\})$ need not be normal. But we did not provide an example there in favor of the statement. So we are now providing below an example to substantiate that claim.

Example 1. For $a, b \in \mathbb{R}$, with $b>a$ we define

$$
\begin{aligned}
\mathscr{P}_{1}= & \{\varnothing, \mathbb{R}\} \cup\{(-\infty, a],(-\infty, b],(a, \infty),[b, \infty),\{b\}, \\
& (-\infty, a] \cup[b, \infty),(-\infty, a] \cup\{b\},(a, b]\}, \\
\mathscr{P}_{2}= & \{\varnothing, \mathbb{R}\} \cup\{(-\infty, a],(a, \infty)\} .
\end{aligned}
$$

The bitopological space $\left(\mathbb{R}, \mathscr{P}_{1}, \mathscr{P}_{2}\right)$ is strongly pairwise normal but the space $\left(\mathbb{R}, \mathscr{P}_{1}\right)$ is not normal.

For brevity, we agree to say a bitopological space $\left(X, \mathscr{P}_{1}, \mathscr{P}_{2}\right)$ is star pairwise normal if $X$ is pairwise normal, and if both the topological spaces $\left(X, \mathscr{P}_{1}\right)$ and $\left(X, \mathscr{P}_{2}\right)$ are normal.

By Example 1, we follow that a strongly pairwise normal space may not be star pairwise normal.

Example 2. Let $a \in \mathbb{R}$. We define

$$
\begin{aligned}
& \mathscr{P}_{1}=\{\varnothing, \mathbb{R}\} \cup\{(-\infty, a],(-\infty, a),(a, \infty), \mathbb{R}-\{a\}\}, \\
& \mathscr{P}_{2}=\{\varnothing, \mathbb{R}\} \cup\{(-\infty, a),[a, \infty),(a, \infty), \mathbb{R}-\{a\}\} .
\end{aligned}
$$

The bitopological space $\left(\mathbb{R}, \mathscr{P}_{1}, \mathscr{P}_{2}\right)$ is star pairwise normal but the space is not strongly pairwise normal. 
In view of Example 1 and Example 2, it follows that the notions of strongly pairwise normality and star pairwise normality are independent.

Theorem 3. Let $\left(X, \mathscr{P}_{1}, \mathscr{P}_{2}\right)$ be strongly pairwise normal. If for each pair of disjoint $\left(\mathscr{P}_{i}\right)$ closed sets $E, F$ there exists a $\left(\mathscr{P}_{j}\right)$ closed set $A$ such that either $E \cap A=\varnothing$ and $F \subset A$ or $F \cap A=\varnothing$ and $E \subset A$, then $\left(X, \mathscr{P}_{i}\right)$ is normal.

Proof. Easy to verify.

Theorem 4. If $A$ is a $\left(\mathscr{P}_{i}\right)$ closed set and $B$ is a $\left(\mathscr{P}_{j}\right)$ closed set in a strongly pairwise normal bitopological space $\left(X, \mathscr{P}_{1}, \mathscr{P}_{2}\right)$ such that $A \cap B=\varnothing$, then there exist a $\left(\mathscr{P}_{i}, \mathscr{P}_{j}\right)$ dually open set $U$ and a $\left(\mathscr{P}_{j}, \mathscr{P}_{i}\right)$ dually open set $V$ such that $A \subset U, B \subset V$ and $U \cap V=\varnothing$.

Proof. Easy to follow from the definition of strong pairwise normality.

Converse of Theorem 4 is not true.

Example 3 (Bose and Mukharjee, [1]). For $a \in \mathbb{R}$, we define

$$
\mathscr{P}_{1}=\{\varnothing, \mathbb{R},(-\infty, a],(a, \infty)\}, \quad \mathscr{P}_{2}=\{\varnothing, \mathbb{R},(-\infty, a),[a, \infty)\}
$$

In the bitopological space $\left(\mathbb{R}, \mathscr{P}_{1}, \mathscr{P}_{2}\right),(a, \infty)$ and $(-\infty, a)$ are only disjoint pair of a $\left(\mathscr{P}_{1}\right)$ closed set and a $\left(\mathscr{P}_{2}\right)$ closed set, respectively, as well as $\left(\mathscr{P}_{1}, \mathscr{P}_{2}\right)$ dually open and $\left(\mathscr{P}_{2}, \mathscr{P}_{1}\right)$ dually open, respectively. So it follows that the bitopological space $\left(\mathbb{R}, \mathscr{P}_{1}, \mathscr{P}_{2}\right)$ satisfies all conditions of Theorem 4 but the space is not strongly pairwise normal.

Theorem 5. A bitopological space $X$ is strongly pairwise normal iff for a $\left(\mathscr{P}_{j}\right)$ closed set $F$ and a $\left(\mathscr{P}_{i}\right)$ open set $U$ with $F \subset U$, there exists a $\left(\mathscr{P}_{i}\right)$ open set $G$ such that $F \subset$ $\left(\mathscr{P}_{j}\right) \operatorname{Int}(G) \subset G \subset\left(\mathscr{P}_{i}\right) \operatorname{Cl}\left(\left(\mathscr{P}_{j}\right) \mathrm{Cl}(G)\right) \subset U$.

Proof. Firstly, suppose that $X$ is strongly pairwise normal. For the $\left(\mathscr{P}_{j}\right)$ closed set $F$ and $\left(\mathscr{P}_{i}\right)$ open set $U$ with $F \subset U$, we obtain a $\left(\mathscr{P}_{i}\right)$ open set $G$ and a $\left(\mathscr{P}_{j}\right)$ open set $H$ such that $F \subset\left(\mathscr{P}_{j}\right) \operatorname{Int}(G), X-U \subset\left(\mathscr{P}_{i}\right) \operatorname{Int}(H)$ and $G \cap H=\varnothing$. $G \cap H=\varnothing$ implies that $G \subset X-H \subset X-\left(\mathscr{P}_{i}\right) \operatorname{Int}(H)$. Also $X-U \subset\left(\mathscr{P}_{i}\right) \operatorname{Int}(H) \subset H$ which in turn implies that $X-H \subset X-\left(\mathscr{P}_{i}\right) \operatorname{Int}(H) \subset U$. So we have $F \subset\left(\mathscr{P}_{j}\right) \operatorname{Int}(G) \subset$ $G \subset\left(\mathscr{P}_{i}\right) \mathrm{Cl}\left(\left(\mathscr{P}_{j}\right) \mathrm{Cl}(G)\right) \subset\left(\mathscr{P}_{i}\right) \mathrm{Cl}\left(\left(\mathscr{P}_{j}\right) \mathrm{Cl}(X-H)\right)=\left(\mathscr{P}_{i}\right) \mathrm{Cl}(X-H) \subset\left(\mathscr{P}_{i}\right) \mathrm{Cl}(X-$ $\left.\left(\mathscr{P}_{i}\right) \operatorname{Int}(H)\right)=X-\left(\mathscr{P}_{i}\right) \operatorname{Int}(H) \subset U$. Thus we get a $\left(\mathscr{P}_{i}\right)$ open set $G$ such that $F \subset$ $\left(\mathscr{P}_{j}\right) \operatorname{Int}(G) \subset G \subset\left(\mathscr{P}_{i}\right) \mathrm{Cl}\left(\left(\mathscr{P}_{j}\right) \mathrm{Cl}(G)\right) \subset U$.

Converse part follows easily.

Theorem 6. Let $\left(X, \mathscr{P}_{1}, \mathscr{P}_{2}\right)$ be a bitopological space and $Y \subset X$. Suppose that for each disjoint pair of a $\left(\mathscr{P}_{i Y}\right)$ closed set $E_{Y}$ and a $\left(\mathscr{P}_{j Y}\right)$ closed set $F_{Y},(i, j \in\{1,2\}, i \neq j)$, there exists a disjoint pair of a $\left(\mathscr{P}_{i}\right)$ closed set $E_{X}$ and a $\left(\mathscr{P}_{j}\right)$ closed set $F_{X}$ such that $E_{Y}=Y \cap E_{X}, F_{Y}=Y \cap F_{X}$. Then the relative bitopological space $\left(Y, \mathscr{P}_{1 Y}, \mathscr{P}_{2 Y}\right)$ is strongly pairwise normal if $\left(X, \mathscr{P}_{1}, \mathscr{P}_{2}\right)$ is strongly pairwise normal.

Proof. Since the bitopological space $\left(X, \mathscr{P}_{1}, \mathscr{P}_{2}\right)$ is strongly pairwise normal, for the disjoint pair of a $\left(\mathscr{P}_{i}\right)$ closed set $E_{X}$ and a $\left(\mathscr{P}_{j}\right)$ closed set $F_{X}$, there exist a $\left(\mathscr{P}_{j}\right)$ open set $G_{X}$ and a $\left(\mathscr{P}_{i}\right)$ open set $H_{X}$ such that $E_{X} \subset\left(\mathscr{P}_{i}\right) \operatorname{Int}\left(G_{X}\right), F_{X} \subset\left(\mathscr{P}_{j}\right) \operatorname{Int}\left(H_{X}\right)$ and $G_{X} \cap H_{X}=\varnothing$. Now

$$
\begin{aligned}
& Y \cap E_{X} \subset Y \cap\left(\mathscr{P}_{i}\right) \operatorname{Int}\left(G_{X}\right) \subset Y \cap G_{X} \\
\Rightarrow \quad & E_{Y} \subset\left(\mathscr{P}_{i Y}\right) \operatorname{Int}\left(Y \cap G_{X}\right)\left(\text { since } Y \cap\left(\mathscr{P}_{i}\right) \operatorname{Int}\left(G_{X}\right) \text { is }\left(\mathscr{P}_{i Y}\right) \text { open }\right) .
\end{aligned}
$$


and

$$
\begin{aligned}
& Y \cap F_{X} \subset Y \cap\left(\mathscr{P}_{j}\right) \operatorname{Int}\left(H_{X}\right) \subset Y \cap H_{X} \\
\Rightarrow \quad & \left.F_{Y} \subset\left(\mathscr{P}_{j Y}\right) \operatorname{Int}\left(Y \cap G_{X}\right) \text { (since } Y \cap\left(\mathscr{P}_{j}\right) \operatorname{Int}\left(H_{X}\right) \text { is }\left(\mathscr{P}_{j Y}\right) \text { open }\right) .
\end{aligned}
$$

Note that $Y \cap G_{X}$ and $Y \cap H_{X}$ are $\left(\mathscr{P}_{j Y}\right)$ open and $\left(\mathscr{P}_{i Y}\right)$ open, respectively, and $\left(Y \cap G_{X}\right) \cap$ $\left(Y \cap H_{X}\right)=\varnothing$ as $G_{X} \cap H_{X}=\varnothing$. So $\left(Y, \mathscr{P}_{1 Y}, \mathscr{P}_{2 Y}\right)$ is strongly pairwise normal.

Choosing $Y=(-\infty, b]$ in Example 1, we see that the relative bitopological space $\left(Y, \mathscr{P}_{1 Y}\right.$, $\left.\mathscr{P}_{2 Y}\right)$ of $\left(\mathbb{R}, \mathscr{P}_{1}, \mathscr{P}_{2}\right)$ satisfies all conditions of Theorem 6 . We see that the relative bitopological space $\left(Y, \mathscr{P}_{1 Y}, \mathscr{P}_{2 Y}\right)$ of the example is strongly pairwise normal which is the desired result according to Theorem 6 as $\left(\mathbb{R}, \mathscr{P}_{1}, \mathscr{P}_{2}\right)$ is strongly pairwise normal.

Remark 1. In topological spaces, we know that closed subspaces of normal spaces are normal. But in Theorem 4, we note that $Y$ need not be closed for any $i \in\{1,2\}$ to be the relative bitopological space $\left(Y, \mathscr{P}_{1 Y}, \mathscr{P}_{2 Y}\right)$ strongly pairwise normal.

Theorem 7. For $Y \subset X$, the relative bitopological space $\left(Y, \mathscr{P}_{1 Y}, \mathscr{P}_{2 Y}\right)$ is strongly pairwise regular if $\left(X, \mathscr{P}_{1}, \mathscr{P}_{2}\right)$ is strongly pairwise regular.

Proof. Similar to that of Theorem 6.

Acknowledgement. The authors are grateful to the referee for providing some constructive suggestions towards the improvement of the paper.

\section{REFERENCES}

1. M.K. Bose, A. Mukharjee, On bitopological full normality, Mat. Vesnik, 62 (2010), 11-18.

2. J.C. Kelly, Bitopological spaces, Proc. Lond. Math. Soc., 13 (1963), №3, 71-89.

3. A. Mukharjee, Some new bitopological notions, Publ. Inst. Math. (Beograd) (N. S.), 93 (2013), $165-172$.

Department of Mathematics

St. Joseph's College, Darjeeling, W. Bengal

ajoyjee@gmail.com

Department of Mathematics

Malda College, Malda, W. Bengal

roychoudhuryarup@yahoo.co.in 